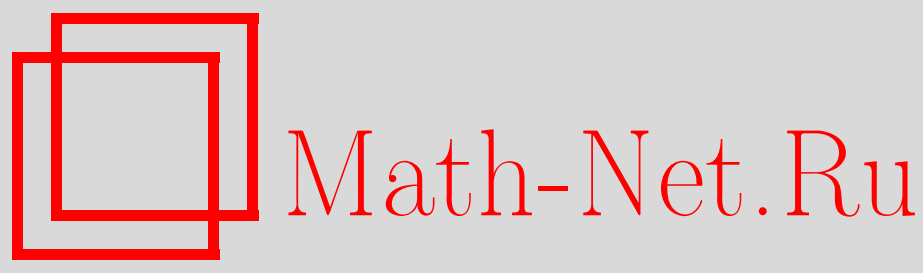

В. И. Денисов, Развитие концепции естественной геометрии для физических взаимодействий, ТМФ, 2017, том 191, номер 2, 205-211

DOI: https://doi.org/10.4213/tmf9182

Использование Общероссийского математического портала Math-Net.Ru подразумевает, что вы прочитали и согласны с пользовательским соглашением http://www . mathnet.ru/rus/agreement

Параметры загрузки:

IP : 54.157 .27 .8

26 апреля 2023 г., 13:57:55

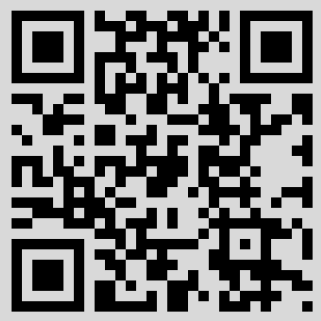




\title{
ФИЗИКА
}

Том 191, № 2

май, 2017

\section{РАЗВИТИЕ КОНЦЕПЦИИ ЕСТЕСТВЕННОЙ ГЕОМЕТРИИ ДЛЯ ФИЗИЧЕСКИХ ВЗАИМОДЕЙСТВИЙ}

\begin{abstract}
Проанализировано развитие концепции естественной геометрии для гравитационного поля в работах Логунова. Обсуждается применение этой концепции к нелинейной электродинамике вакуума. Показано, что определение естественной геометрии для нелинейной теории и нахождение ее метрического тензора позволяет получать достаточно полную информацию о законах распространения импульсов электромагнитного поля во внешних электромагнитных полях.
\end{abstract}

Ключевые слова: естественная геометрия, метрический тензор, гравитационное поле, нелинейная электродинамика вакуума.

DOI: https://doi.org/10.4213/tmf9182

В работах академика Логунова большое внимание уделялось вопросам теории гравитации и, особенно, выбору естественной геометрии для гравитационного поля и других физических полей. Вопрос о естественной геометрии для физических взаимодействий впервые был сформулирован Лобачевским в 1829 г. В своей работе [1] он писал: “... не может быть никакого противоречия, когда мы допускаем, что некоторые силь в природе следуют одной, другие своей особой Геометрии".

Однако до начала XX века эта концепция не получила своего развития, так как все существовавшие теоретико-полевые модели физических взаимодействий использовали геометрии Евклида или Минковского. Ситуация изменилась после создания Эйнштейном [2] общей теории относительности (ОТО), столетний юбилей которой мы отметили в 2015 г. Эта теория внесла в физику представление о том, что естественной геометрией для гравитационного поля и всех полей материи является псевдориманова геометрия, метрический тензор которой согласно уравнениям Эйнштейна предопределяется распределением материи в пространстве. В ОТО гравитационное поле отождествляется с метрическим тензором пространства-времени $g_{i k}$, и это поле проявляется в искривлении пространства-времени.

В работах Логунова [3]-[5] развивался иной подход, в котором гравитационное поле рассматривалось как физическое поле в духе Фарадея-Максвелла. В этом

* Московский государственный университет им. М. В. Ломоносова, Москва, Россия. E-mail: denisov@srd.sinp.msu.ru 
подходе полевой переменной для описания гравитации служил симметричный тензор второго ранга $\varphi_{i k}=\varphi_{k i}$, и естественной геометрией для этого поля являлась псевдоевклидова геометрия с метрическим тензором $\gamma_{i k}$.

Универсальный характер воздействия гравитационного поля на вещество, проявляющийся в равенстве инертной и гравитационной масс любого тела, требовал, чтобы движение вещества, взаимодействующего с внешним гравитационным полем, было локально неотличимым от свободного движения вещества в соответствующей ускоренной системе отсчета. Следствием этой универсальности является необходимость геометризации плотности лагранжиана вещества. Развивая эту идею, Логунов в дальнейших работах [6], [7] сформулировал принцип тождественности (принцип геометризации), согласно которому уравнения движения вещества под действием гравитационного поля $\varphi_{i k}$ в псевдоевклидовом пространстве-времени с метрическим тензором $\gamma_{i k}$ можно тождественно представить как уравнения движения вещества в римановом пространстве-времени с метрическим тензором $g_{i k}$, зависящим от гравитационного поля $\varphi_{i k}$ и метрического тензора $\gamma_{i k}$. В соответствии с принципом тождественности геометризация теории проводилась в той минимальной степени (в виде геометризации плотности лагранжиана вещества), которая позволила описать известные экспериментальные факты и сохранить общепринятые понятия энергии-импульса для системы, включающей вещество и гравитационное поле.

Для определения явной зависимости метрического тензора $g_{i k}$ от $\gamma_{i k}$ и $\varphi_{i k}$ использовались уравнения

$$
\frac{\partial g_{n m}}{\partial \varphi_{i k}}=\frac{1}{2}\left[\delta_{m}^{i} \delta_{n}^{k}+\delta_{m}^{k} \delta_{n}^{i}\right]
$$

которые имели вытекающий из выполненных гравитационных экспериментов постньютоновский предел.

Дальнейшее развитие этой теории привело к созданию теории гравитации [8]-[12], в которой гравитон обладал массой $m \sim 10^{-66}$ г. Уравнения гравитационного поля в теории с массивным гравитоном имеют вид

$$
R^{i k}-\frac{1}{2} g^{i k} R+\frac{m^{2}}{2}\left[g^{i k}+\left(g^{i m} g^{k n}-\frac{1}{2} g^{i k} g^{n m}\right) \gamma_{n m}\right]=8 \pi T^{i k} .
$$

Метрический тензор псевдориманова пространства-времени $g_{i k}$ в этой теории связан с гравитационным полем $\varphi_{i k}$ и метрическим тензором псевдоевклидова пространства-времени $\gamma_{i k}$ соотношением

$$
\tilde{g}^{i k}=\tilde{\gamma}^{i k}+\widetilde{\varphi}^{i k}
$$

где

$$
\tilde{g}^{i k}=\sqrt{-g} g^{i k}, \quad \tilde{\gamma}^{i k}=\sqrt{-\gamma} \gamma^{i k}, \quad \widetilde{\varphi}^{i k}=\sqrt{-\gamma} \varphi^{i k} .
$$

Как показано в работе [7], теория с уравнениями (1), (2) описывает все гравитационные эксперименты, выполненные в Солнечной системе с постньютоновской точностью.

Концепция естественной геометрии для физических взаимодействий нашла свое применение не только в гравитации, но и в нелинейной электродинамике вакуума. В настоящее время в научной литературе [13]-[15] рассматриваются несколько теоретических моделей нелинейной электродинамики вакуума. В этих теориях нелинейность уравнений существенно проявляется при напряженностях электромагнитных полей $B, E \sim B_{\mathrm{q}}$, где $B_{\mathrm{q}}=4.41 \cdot 10^{13}$ Гс. 
В случаях, когда $B, E<B_{\mathrm{q}}$, лагранжианы этих теорий можно записать в параметризованном постмаксвелловском приближении [16]-[18]:

$$
L=\frac{\sqrt{-g}}{32 \pi}\left\{2 F_{(2)}+\xi\left[\left(\eta_{1}-2 \eta_{2}\right) F_{(2)}^{2}+4 \eta_{2} F_{(4)}\right]+O\left(\xi^{2} F_{2}^{3}\right)\right\}-\frac{\sqrt{-g}}{c} j^{k} A_{k},
$$

где $\xi=1 / B_{\mathrm{q}}^{2}, F_{(2)}=F_{n m} F^{m n}$ и $F_{(4)}=F_{m n} F^{n i} F_{i k} F^{k m}$ - инварианты тензора электромагнитного поля $F_{n m}$, индексы у которых поднимаются и опускаются с помощью метрического тензора псевдориманова пространства-времени $g_{i k}, \eta_{1}$ и $\eta_{2}$ - постмаксвелловские параметры, которые в электродинамике Борна-Инфельда совпадают, $\eta_{1}=\eta_{2}$, а в теории Гейзенберга-Эйлера различаются:

$$
\eta_{1}=\frac{\alpha}{45 \pi}=5.1 \cdot 10^{-5}, \quad \eta_{2}=\frac{7 \alpha}{180 \pi}=9.0 \cdot 10^{-5}
$$

Уравнения электромагнитного поля нелинейной электродинамики с лагранжианом (3):

$$
\frac{1}{\sqrt{-g}} \frac{\partial}{\partial x^{n}}\left\{\sqrt{-g}\left[\left(1+\xi\left(\eta_{1}-2 \eta_{2}\right) F_{2}\right) F^{m n}+4 \xi \eta_{2} F^{m l} F_{l k} F^{k n}\right]\right\}=-\frac{4 \pi}{c} j^{m},
$$

вместе с системой однородных уравнений

$$
\frac{\partial F_{i k}}{\partial x^{n}}+\frac{\partial F_{k n}}{\partial x^{i}}+\frac{\partial F_{n i}}{\partial x^{k}}=0
$$

представляют собой систему нелинейных дифференциальных уравнений в частных производных первого порядка, к которым методы интегрирования линейных уравнений [19] неприменимы. Очевидно, что эту систему уравнений можно точно решить лишь в небольшом числе простейших физических ситуаций (см., например, статьи [20]-[22]).

Для интегрирования системы уравнений (4), (5) в ряде работ (см., например, [23], [24]) был разработан модифицированный метод, основанный на методе НьюменаПенроуза [25], хорошо себя зарекомендовавший при решении задач ОТО. Однако из-за большой эвристичности этого метода добиться существенного успеха и на этом пути не удалось. Поэтому определение естественной геометрии для электромагнитного поля в нелинейной электродинамике вакуума и нахождение метрического тензора этой геометрии дает возможность теоретического исследования основных закономерностей электромагнитных явлений в этой теории.

Как указывалось в книге Логунова [26], “... любому физическому полю соответствует некоторая геометрия, называемая естественной, именно такая, что в отсутствие взаимодействия с другими полями фронт свободной волны этого физического поля движется по геодезической естественного пространства-времени". Поэтому для нахождения метрического тензора естественной геометрии для электромагнитного поля в нелинейной электродинамике вакуума необходимо построить уравнение фронта волны (уравнение характеристик) для системы уравнений (4), (5). Для линейной электродинамики в произвольной материальной среде оно было получено в работах [27], [28] и имело следующий вид:

$$
C^{i k n m}\left(\frac{\partial S}{\partial x^{i}}\right)\left(\frac{\partial S}{\partial x^{k}}\right)\left(\frac{\partial S}{\partial x^{n}}\right)\left(\frac{\partial S}{\partial x^{m}}\right)=0 .
$$


Для нелинейной электродинамики (4), (5) уравнение фронта волны было построено в статьях [29], [30]. В этом случае тензор $C^{i k n m}$ квадрируется, в результате чего уравнение фронта волны принимает вид

$$
G_{(1)}^{i k} G_{(2)}^{n m}\left(\frac{\partial S}{\partial x^{i}}\right)\left(\frac{\partial S}{\partial x^{k}}\right)\left(\frac{\partial S}{\partial x^{n}}\right)\left(\frac{\partial S}{\partial x^{m}}\right)=0 .
$$

При $\eta_{1} \neq \eta_{2}$ в этом уравнении присутствуют два различных метрических тензора $G_{(1)}^{i k}$ и $G_{(2)}^{n m}$ естественной геометрии для электромагнитного поля:

$$
\begin{gathered}
G_{(1)}^{i k}=g^{i k}+4 \eta_{1} \xi F^{i p} F_{p^{\cdot}}^{\cdot k}, \\
G_{(2)}^{n m}=g^{n m}+4 \eta_{2} \xi F^{n l} F_{l \cdot}^{\cdot m} .
\end{gathered}
$$

Таким образом, при $\eta_{1} \neq \eta_{2}$ закон распространения электромагнитной волны во внешнем электромагнитном поле зависит от поляризации волны (двулучепреломление). При этом мы имеем две нормальные волны, первая из которых распространяется по геодезическим псевдориманова пространства-времени с метрическим тензором $G_{(1)}^{i k}$, а вторая, имеющая ортогональную поляризацию, - по изотропным геодезическим с метрическим тензором $G_{(2)}^{n m}$. Метрические тензоры $G_{(1)}^{i k}$ и $G_{(2)}^{n m}$ естественной геометрии в нелинейной электродинамике вакуума зависят не только от метрического тензора $g^{n m}$, получаемого из уравнений гравитационного поля, но и от квадратичной комбинации тензора электромагнитного поля. Однако, как показано в работе [31], это не означает, что в нелинейной электродинамике вакуума принцип эквивалентности Эйнштейна для фотонов [32] нарушается. Наличие в метрическом тензоре естественной геометрии квадратичной комбинации тензора электромагнитного поля означает, что в нелинейной электродинамике вакуума на распространение фотонов влияет не только гравитационное поле, но и внешнее электромагнитное поле.

Знание метрических тензоров $G_{(1)}^{i k}$ и $G_{(2)}^{n m}$ позволяет, не обращаясь к нелинейным уравнениям (4), решать широкий круг задач о распространении электромагнитных волн во внешних электромагнитных полях. Это и задачи об искривлении лучей электромагнитных волн во внешних неоднородных электромагнитных полях [33], и задачи о запаздывании во внешнем неоднородном поле электромагнитного импульса, переносимого первой нормальной волной, по сравнению с импульсом, переносимым другой нормальной волной, имеющей ортогональную поляризацию [34]-[37], а также задачи нелинейной лазерной физики [38], [39].

Действительно, если уравнения

$$
G_{(1,2)}^{i k}\left(\frac{\partial S}{\partial x^{i}}\right)\left(\frac{\partial S}{\partial x^{k}}\right)=0
$$

позволяют провести разделение переменных, то их можно решить методом Гамильтона-Якоби. Это дает возможность записать уравнения для лучей, по которым распространяются нормальные волны, и определить закон движения электромагнитных импульсов по этим лучам. Если задача не обладает достаточной симметрией для разделения переменных, то можно воспользоваться методом Лагранжа-Шарпи [40] и перейти от уравнения фронта волны (7) к уравнениям для изотропных геодезических в пространстве-времени с метрическими тензорами $G_{(1)}^{i k}$ и $G_{(2)}^{i k}[41]$ :

$$
\frac{d k^{n}}{d \sigma}=-G_{(1,2)}^{n p}\left[\frac{\partial G_{p m}^{(1,2)}}{\partial x^{i}}-\frac{1}{2} \frac{\partial G_{m i}^{(1,2)}}{\partial x^{p}}\right] k^{i} k^{m}=0,
$$


где $\sigma$ - некоторый аффинный параметр, $k^{n}=d x^{n} / d \sigma$ - касательный вектор к геодезической, удовлетворяющий условию

$$
G_{n k}^{(1,2)} \frac{d x^{n}}{d \sigma} \frac{d x^{k}}{d \sigma}=0 .
$$

Так как в большинстве лабораторных и астрофизических ситуаций (при $B, E<B_{\mathrm{q}}$, где $\left.B_{\mathrm{q}}=4.41 \cdot 10^{13} \Gamma \mathrm{c}\right)$ правая часть уравнений (8) содержит малый параметр, систему уравнений (8) можно решить методом последовательных приближений.

Следует отметить, что нахождение уравнения фронта волны и определение естественной геометрии позволяет решать широкий круг задач и в нелинейной электродинамике сплошных сред.

Таким образом, концепция естественной геометрии для физических взаимодействий наиболее плодотворна для нелинейных теорий, в которых нелинейность содержится при старших производных в лагранжиане.

\section{Список литературы}

[1] Н. И. Лобачевский, "О началах геометрии”, Казанский Вестник, XXV (1829), 178-187.

[2] A. Einstein, "Die Feldgleichungen der Gravitation", Sitzungsber. Königl. Preuß. Akad. Wiss. (Berlin), 48 (1915), 844-847.

[3] А.А. Логунов, В.Н. Фоломешкин, “Энергия-импульс гравитационных волн в общей теории относительности", ТМФ, 32:2 (1977), 167-175.

[4] А. А. Логунов, В. Н. Фоломешкин, "Проблемы энергии-импульса и теория гравитации", TMФ, 32:3 (1977), 291-325.

[5] А. А. Логунов, В.Н. Фоломешкин, "О геометризованных теориях гравитации”, ТМФ, 32:2 (1977), 147-166.

[6] А. А. Логунов, В. И. Денисов, А. А. Власов, М. А. Мествиришвили, В. Н. Фоломешкин, "Новые представления о пространстве-времени и гравитации", ТМФ, 40:3 (1979), 291-328.

[7] A. A. Logunov, M.A. Mestvirishvili, "Relativistic theory of gravitation", Prog. Theor. Phys., 74:1 (1985), 31-50.

[8] А.А. Логунов, “Основные принципы релятивистской теории гравитации”, ТМФ, 80:2 (1989), 165-172.

[9] А.А. Логунов, “Основные уравнения для массивного гравитационного поля", ТМФ, 92:2 (1992), 191-206.

[10] С. С. Герштейн, А. А. Логунов, М. А. Мествиришвили, "Самоограничение гравитационного поля и его роль во Вселенной”, УФН, 176:11 (2006), 1207-1225.

[11] С. С. Герштейн, А. А. Логунов, М.А. Мествиришвили, "Гравитационные волны в релятивистской теории гравитации", ТМФ, 160:2 (2009), 270-275.

[12] А. А. Логунов, Релятивистская теория гравитации, Наука, М., 2012.

[13] M. Born, L. Infeld, "Foundations of the new field theory", Proc. Roy. Soc. Ser. A, 144:852 (1934), 425-430.

[14] W. Heisenberg, H. Euler, "Folgerungen aus der Diracschen Theorie des Positrons", Z. Phys., 98:11 (1936), 714-720.

[15] G. Boillat, "Nonlinear electrodynamics: Lagrangians and equations of motion", J. Math. Phys., 11:3 (1970), 941-950.

[16] В. И. Денисов, И. П. Денисова, "Проверяемый пост-максвелловский эффект нелинейной электродинамики в вакууме”, Оптика и спектроскопия, 90:2 (2001), 329-335. 
[17] В. И. Денисов, И.П. Денисова, "Взаимодействие интенсивного лазерного излучения со слабыми электромагнитными волнами на вакуумном участке кольцевого лазера", Оптика и спектроскопия, 90:6 (2001), 1023-1026.

[18] I. P. Denisova, I. V. Krivchenkov, P. A. Vshivtseva, A. A. Zubrilo, "Letter: Nonlinear gravitational-electromagnetic bending of the rays of weak electromagnetic waves in the fields of pulsars and magnetars", Gen. Relativ. Gravitation, 36:4 (2004), 889-897.

[19] К. В. Жуковский, “Метод обратных дифференциальных операторов с использованием ортогональных полиномов и специальных функций для решения некоторых типов дифференциальных уравнений и физических задач", Вестн. Моск. ун-та. Сер. 3. Физ. Астрон., 2015, № 2, 19-26.

[20] Б. М. Барбашов, Н.А. Черников, "Решение задачи о рассеянии двух плоских волн в нелинейной скалярной теории поля типа Борна-Инфельда", ЖЭТФ, 51:8 (1966), $658-668$.

[21] В. И. Денисов, И. П. Денисова, "Эффект взаимодействия плоских электромагнитных волн в нелинейной электродинамике Борна-Инфельда", ТМФ, 129:1 (2001), 131-139.

[22] В.И. Денисов, В.А. Соколов, "Исследование регуляризующих свойств нелинейной электродинамики в теории Эйнштейна-Борна-Инфельда", ЖЖЭТФ, 140:6 (2011), 1064.

[23] И. П. Денисова, "Развитие метода спиновых коэффициентов для интегрирования уравнений биметрических теорий гравитации”, Дифферени. уравнения, 35:7 (1999), 935-941.

[24] П. А. Вшивцева, А. А. Зубрило, И.В.Кривченков, В. А. Соколов, "Развитие метода спиновых коэффициентов для интегрирования уравнений нелинейной электродинамики вакуума", Вестн. Моск. ун-та. Сер. 3. Физ. Астрон., 2006, № 5, 6-8.

[25] Г. А. Алексеев, В.И. Хлебников, "Формализм Ньюмена-Пенроуза и его применение в общей теории относительности", ЭЧАЯ, 9:5 (1978), 790-870.

[26] А. А. Логунов, Лекиии по теории относительности и гравитации, Наука, М., 1987.

[27] Р. Курант, Уравнения с частными призводными, Мир, М., 1964.

[28] W.-T. Ni, "Spacetime structure and asymmetric metric from the premetric formulation of electromagnetism", Phys. Lett. A, 379:20-21 (2015), 1297-1303.

[29] В. И. Денисов, И. П. Денисова, "Уравнения эйконала в параметризованной нелинейной электродинамике вакуума", Докл. РАН, 378:4 (2001), 463-465.

[30] В. И. Денисов, "Исследование эффективного пространства-времени нелинейной электродинамики вакуума в поле магнитного диполя", ТМФ, 132:2 (2002), 211-221.

[31] М. Г. Гапочка, М. М. Денисов, И. П. Денисова, Н. В. Каленова, А. Ф. Королев, “Математическое моделирование нелинейно-электродинамического эффекта запаздывания сигналов в магнитном поле пульсаров", Журн. вычисл. матем. и матем. физ., 55:11 (2015), 1893-1903.

[32] V.I. Denisov, M. I. Denisov, Phys. Rev. D, 60:4 (1999), 047301, 4 pp.

[33] J. Y. Kim, "Bending of electromagnetic wave in an ultra-strong magnetic field", J. Cosmol. Astropart. Phys., 10 (2012), 056, 9 pp., arXiv: 1208.1319.

[34] В. И. Денисов, И. В. Кривченков, И. П. Денисова, "Эффект нелинейно-электродинамического запаздывания электромагнитных сигналов в поле магнитного диполя", ЖЭТФ, 122:2(8) (2002), 227-232.

[35] V.I. Denisov, S. I. Svertilov, "Nonlinear electromagnetic and gravitational actions of neutron star fields on electromagnetic wave propagation", Phys. Rev. D, 71:6 (2005), 063002, $13 \mathrm{pp}$.

[36] М. И. Васильев, В. А. Соколов, "Нелинейно-электродинамические эффекты в электромагнитном поле вращающегося пульсара", Вестн. Моск. ун-та. Сер. 3. Физ. Астрон., 67:5 (2012), 8-12.

[37] V.I. Denisov, V. A. Sokolov, M. I. Vasili'ev, "Nonlinear vacuum electrodynamics birefringence effect in a pulsar's strong magnetic field", Phys. Rev. D., 90:2 (2014), 023011, 7 pp. 
[38] V.I. Denisov, "Nonlinear effect of quantum electrodynamics for experiments with a ring laser", J. Opt. A, 2:5 (2000), 372-379.

[39] В. А. Соколов, "Взаимодействие электромагнитных волн в сильном постоянном магнитном поле", Вестн. Моск. ун-та. Сер. 3. Физ. Астрон., 2009, № 3, 108-110.

[40] Л.Э. Эльсгольц, Дифференциальные уравнения и вариационное исчисление, Наука, М., 1965.

[41] П. А. Вшивцева, М. М. Денисов, "Математическое моделирование распространения электромагнитных волн в нелинейной электродинамике", Ж. вычисл. матем. и матем. физ., 49:12 (2009), 2189-2200. 